\title{
Rate capability and stability studies on small-Pad resistive Micromegas
}

\author{
M.T. Camerlingo ${ }^{a, b, c *}$ and M. Alviggi ${ }^{d, e}$, V. Canale ${ }^{d, e}$, M. Della Pietra ${ }^{d, e}$, C. Di \\ Donato $^{e, f}$, P. lengo ${ }^{c}$, M. lodice $^{b}$, F. Petrucci ${ }^{a, b}$, G. Sekhniaidze ${ }^{d}$
}

a Univ. Roma Tre,

Via della Vasca Navale 84, Rome, Italy

b INFN Roma Tre,

Via della Vasca Navale 84, Rome, Italy

c CERN

Esplanade des Particules 1, 1211 Meyrin, Switzerland

d Univ. Federico II,

Via Cinthia 21, Naples, Italy

e INFN Napoli

Via Cinthia 21, Naples, Italy

fUniv. Parthenope,

Centro Direzionale, isola C4, Naples, Italy

E-mail: maria.teresa.camerlingo@cern.ch, Mariagrazia.Alviggi@cern.ch, Vincenzo.Canale@cern.ch, Massimo.Della.Pietra@cern.ch, camilla.di.donatolcern.ch, Paolo.Iengodcern.ch, Fabrizio.Petruccidcern.ch, givi.sekhniaidzedcern.ch

Micromegas are among the most promising micro pattern gaseous detector (MPGD) technologies for applications in high energy physics (HEP). Micromegas are very versatile. They can be used for precision tracking and trigger, in particle flow sampling calorimetry, as anode planes for RICH detectors or for time projection chambers. Driven mainly by future upgrades of existing experiments at high-luminosity LHC (HL-LHC) and for applications at future accelerators, we started an R\&D project to push further this technology for operations under very high particle flow up to rates of tens $\mathrm{MHz} / \mathrm{cm}^{2}$, three orders of magnitude higher than current applications. The miniaturization of the readout elements and the optimization of the spark protection system, as well as the stability and robustness under operation, are the primary challenges of the project.

40th International Conference on High Energy physics - ICHEP2020

July 28 - August 6, 2020

Prague, Czech Republic (virtual meeting)

*Speaker

(c) Copyright owned by the author(s) under the terms of the Creative Commons

Attribution-NonCommercial-NoDerivatives 4.0 International License (CC BY-NC-ND 4.0). 


\section{Introduction}

A Micromegas detector is a planar proportional mode micro-pattern gaseous detector [1]. Its active volume is divided in two gaps by a micromesh (see Fig. 1). It physically divides the conversion gap between the cathode plane and micromesh, in which a drift electric field is applied, and the region of signal amplification between the micromesh and the strip-segmented anodic plane, in which another electric field is applied and it is enough intense to allow the phenomenon of electron avalanche multiplication, using a relative low voltage difference between the micromesh and the anode plane at a distance of about $120 \mathrm{um}$. To quench the discharges in the amplification gap, a resistive scheme has been adopted, by adding a layer of resistive strips capacitively coupled with the readout strips in the resistive Micromegas detectors [2].

To efficiently operate at $10 \mathrm{MHz} / \mathrm{cm}^{2}$ particle rates, a mandatory choice was the miniaturization of readout strips in $\mathrm{mm}^{2}$ pads to decrease the occupancy of readout electrodes.

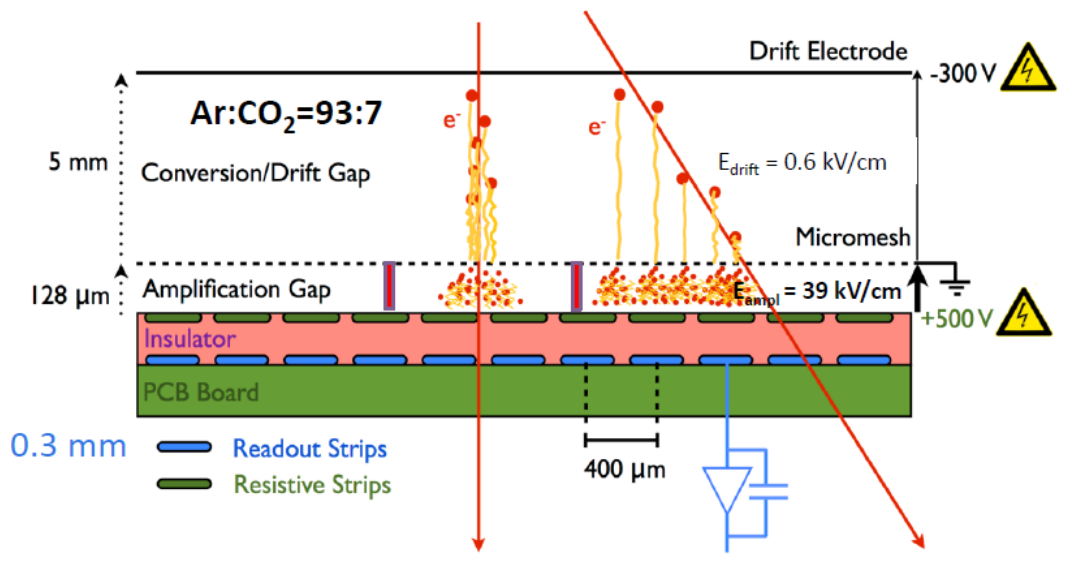

Fig.1: Sketched section of a Micromegas resistive strip detector.

\section{Development of small-Pad resistive Micromegas detectors}

Several small-pad Micromegas detectors have been built with similar anode planes: segmented with a matrix of $48 \times 16$ readout pads with a rectangular shape $\left(0.8 \times 2.8 \mathrm{~mm}^{2}\right)$ and with a pitch of 1 and $3 \mathrm{~mm}$ in the two coordinates, respectively. The active surface is $4.8 \times 4.8 \mathrm{~cm}^{2}$ with 768 electronics channels routed off-detector for readout. Consequently, a new sparks suppression resistive layout is needed to match the pad geometry. Two different schemes were implemented (see Fig. 2-Left and Fig. 2-Right).

The first scheme (Fig. 2-Left) involves a pad-patterned layer with embedded resistors for each readout pad and it will be referred to as PAD-P. The second scheme (Fig. 2-Right) exploits a double layer of Diamond-Like-Carbon (DLC) resistive foils, which sizes are the same as the active area.

Since 2016, long-term characterization and performance studies of these detectors have been carried out. The most complete comparison between the performances of the prototypes with the different resistive layouts are reported in the Reff. [3] and [4].

In all the performed studies, the gas mixture was $93 \% \mathrm{Ar}: 7 \% \mathrm{CO}_{2}$. The PAD-P detector resulted stable and robust against discharges. 
It has a fair spatial resolution of $190 \mu \mathrm{m}$ in the $1 \mathrm{~mm}$ pitch coordinate and a low energy resolution about $48 \%$ (FWHM). The PAD-P gain is affected by the charging-up effect (decreasing by about $20 \%$ at a particle rate of $\sim 1 \mathrm{MHz} / \mathrm{cm}^{2}$ ), due to the exposed dielectrics. This is also visible in the time evolution of the current (Ref. [3]).
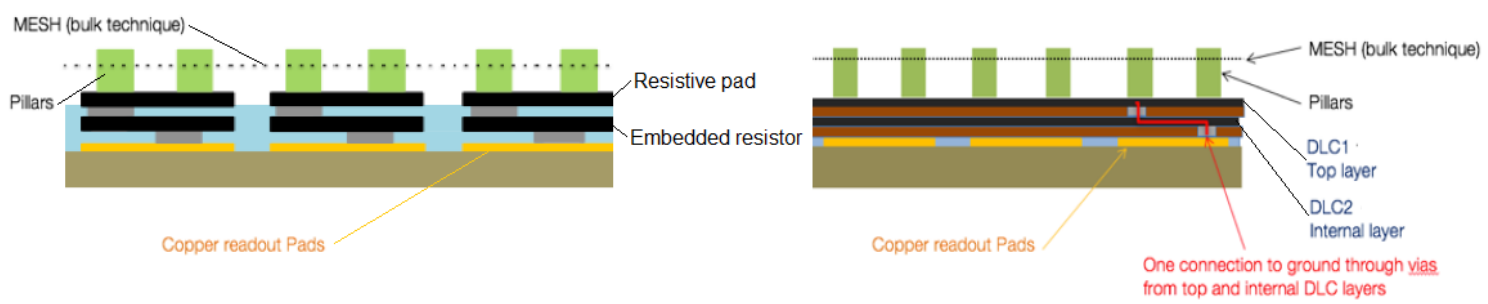

Fig. 2: Sketched transverse section of spark suppression resistive scheme PAD-P (2-Left) and scheme DLC (2-Right) [4].

Trying to improve the performance of PAD-P scheme and following the general interest on the Diamond-Like-Carbon application in MPGD, the second layout was designed like the sketch in Fig. 2-Right, where the pad-patterned planes and intermediated embedded resistors are replaced by two continuous DLC foils. The spatial resolution improves, below $100 \mu \mathrm{m}$ on the same precision coordinate, because of a larger spread of the charge among the pads. The energy resolution is also better thanks to a more uniform electric field, that is not affected by edge effects on the resistive layer pads. The charging-up effect is minimized (Ref. [4]). In this scheme, a net of low resistive silver vias evacuates the current from the double layer of DLC to ground, avoiding the reduction of the rate capability due to the current spread on all the planes. Several DLC prototypes have been built, with different techniques and different values of the average resistivity of the DLC foils [3]. Among those, the best performances are obtained with the DLC20-6 mm vias pitch detector, built with approximately $20 \mathrm{M} \Omega /$ sq resistivity.

\section{Study on the rate capability}

The rate capability is defined by the gain stability with respect to the particle rate variation. Increasing the rate, the gain drops in these detectors, when the current on the resistive elements or charging-up effect become significant. Therefore, the relative gain respect to its value at low rates is studied as a function of the rate to find the rate value at which the gain drops below a set limit (20\%), per different amplification voltages. The relative gain is reported as a function of hit rates using a ${ }^{55} \mathrm{Fe}$ radioactive source (circles markers) to reach the lowest rate values or an X-Ray gun (triangle markers) in the rest of rate range in Fig. 3, for PAD-P and DLC20, as the best representative of DLC family.

PAD-P requires a higher voltage respect to DLC20 to set the same gain factor, about plus $20 \mathrm{~V}$. This may be in part due to the effective characteristics of an individual detector and due to boundary effects in the amplification electric field configuration (to be investigated further). 
In the rate range below $0.5 \mathrm{MHz} / \mathrm{cm}^{2}$, the gain trend is mainly affected by charging-up in PAD-P and its drop is already significant at $540 \mathrm{~V}$ (approximately a gain of $10^{4}$ ) amplification voltage, where the gain at low rate is $\sim 10^{4}$ (black in Fig. 3). Conversely, the gain trend is quite stable in this range in DLC20, where there is not exposed dielectric material in the amplification gap that can charge-up (with the exception of the pillars).

In the full X-Ray rate range, the trend of PAD-P visibly changes above $5 \mathrm{MHz} / \mathrm{cm}^{2}$ because the charging-up and ohmic contributions behave different. The latter becomes in fact more relevant as the rate increase while the charging-up saturates. In the high rate regime, a gain drop of $20 \%$ is reached for PAD-P at approximately $25 \mathrm{MHz} / \mathrm{cm}^{2}$ operating the detector at $530 \mathrm{~V}$ (corresponding to a gain of $\sim 6500$ at low rates). The same drop is reached for DLC20 (at equivalent gain - i.e. $\mathrm{HV}=510 \mathrm{~V}$ ) around $20 \mathrm{MHz} / \mathrm{cm} 2$. At higher rates, PAD-P has a smaller gain drop than DLC20.
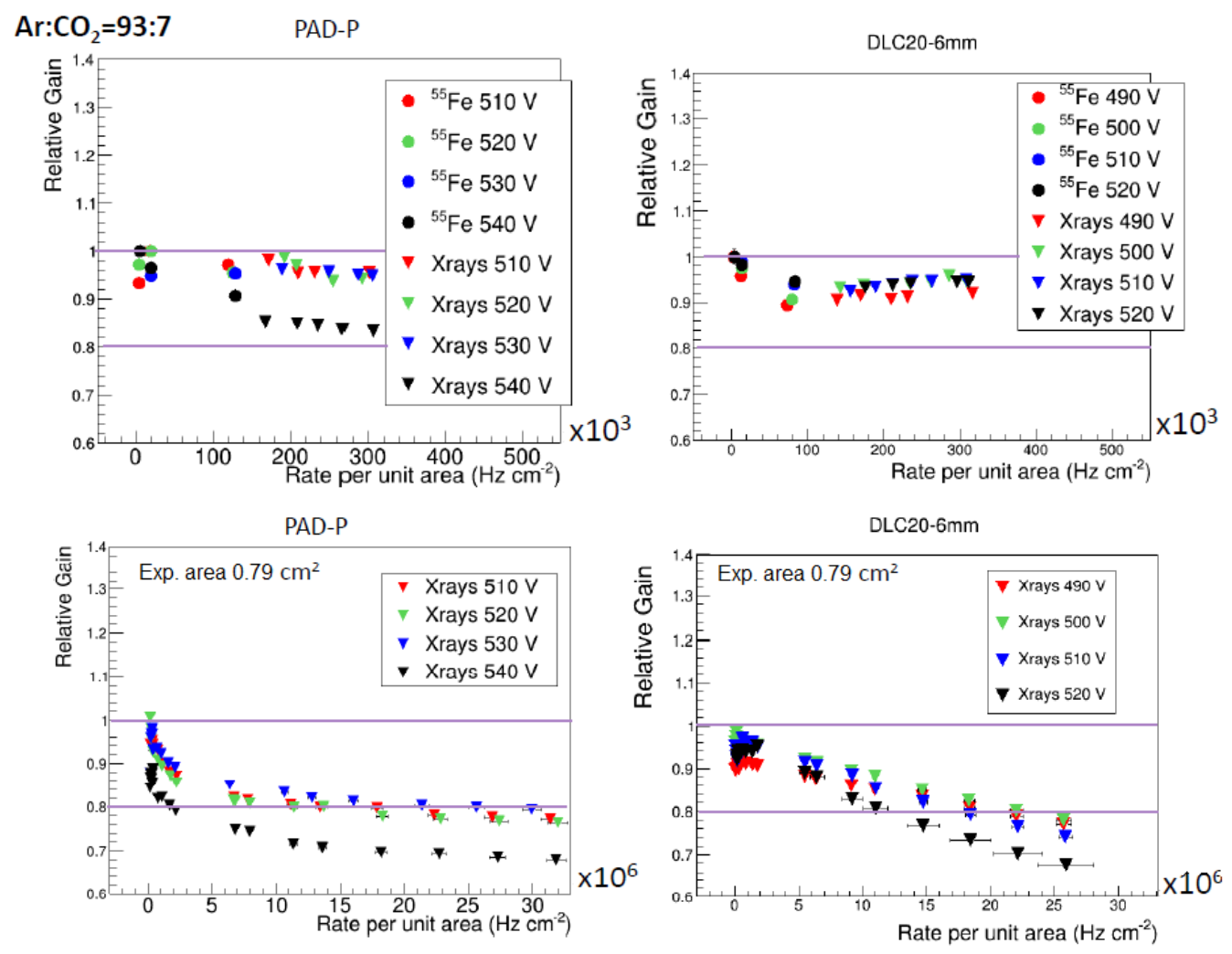

Fig. 3: Relative gain respect to value at low rates as function of rate per unit area $\left({ }^{55} \mathrm{Fe}\right.$ circle markers and X-Ray gun triangle markers).

The study with X-Ray gun is repeated varying the exposure area $\left(0.79 \mathrm{~cm}^{2}\right.$ circle, $3.69 \mathrm{~cm}^{2}$ square, $9 \mathrm{~cm}^{2}$ circle) and reported in Fig. 4. PAD-P gain drop is independent on irradiated area. While, in the DLC prototype, it becomes independent on the irradiated area when irradiated area is significantly larger than the cell defined by the vias pitch. 

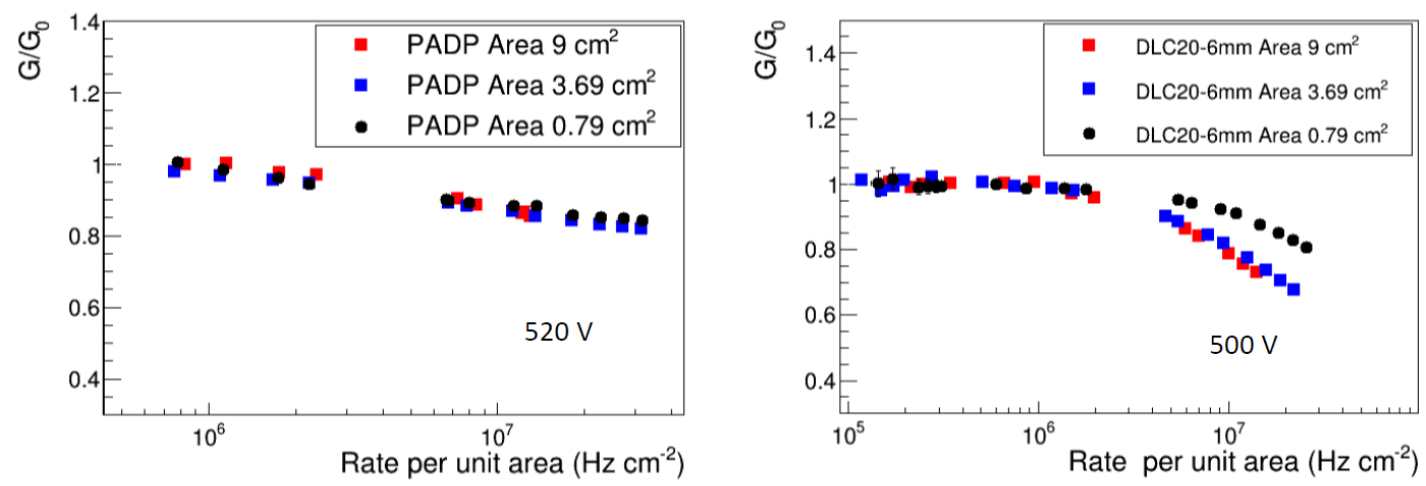

Fig. 4: Relative gain respect to value at low rates as function of rate per unit area for different exposure areas $\left(0.79 \mathrm{~cm}^{2}\right.$ circle, $3.69 \mathrm{~cm}^{2}$ square, $9 \mathrm{~cm}^{2}$ circle) .

\section{Study on stability}

During the test beam at the PSI $\pi \mathrm{M} 1$ beam line $\left(300 \mathrm{MeV} / \mathrm{c}\right.$ charged pion $\left.{ }^{+}\right)$, a first study on stability was carried on. The gain curves in Fig. 5-Left as a function of the amplification were reproduced by the test beam data. They were used to plot the spark rate probability as function of gain. In this study, a current increase was counted as a spark when it is larger than $30 \%$ of the stable value at fixed amplification value. Consequently, the spark probability(/pion/area) was defined as number of sparks per the time acquisition interval, per area and per particle rate (see Fig. 5-Right).
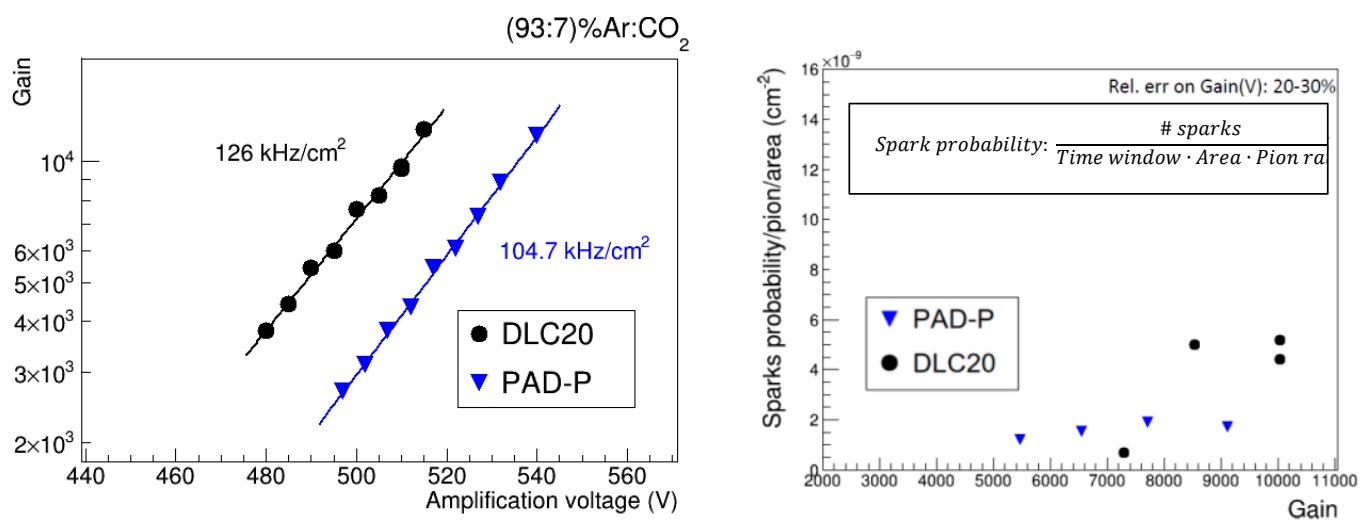

Fig. 5: Gain as function of amplification voltage (Left) and spark rate probability per incident particle (pions) and per unit area as function of gain measured during test beam a $300 \mathrm{MeV} / \mathrm{c}$ charged pion beam (Right).

As already anticipated, PAD-P is the most robust prototypes (see Fig. 5-Right). From this study and all previous tests, the PAD-P detector shows a very good stability and the possibility to be operated at gains larger then $10^{4}$. The DLC20 has also a low spike probability, lower than $5 \times 10^{-9}$ $\mathrm{cm}^{-2}$ per incident pion up to a gain of $10^{4}$. However, if it operates at gains larger than $10^{4}$, its HV behaviour could be subject significant instabilities. Moreover, the comparison between PAD-P and the first DLC prototypes is not conclusive because low resistance spots were found in the 
DLC prototypes during their characterization tests. They consist in some current evacuation silver vias that are in part directly exposed to the charges in the amplification gap.

\section{Conclusions}

For particle rate larger than $10 \mathrm{MHz} / \mathrm{cm}^{2}$ the PAD read-out geometry requires a new resistive layout. Two different layouts were implemented in several prototypes, most of them are based on DLC foils. The performances of the detectors were compared in similar gas and gain conditions. The general conclusion is that both layouts are valid spark suppression resistive systems for operations up to tens $\mathrm{MHz} / \mathrm{cm}^{2}$.

The so far conducted studies on the rate capability and the preliminary studies on stability confirm that PAD-P has an excellent robustness and stability at gains higher than $10^{4}$ under high irradiation. The first DLC prototypes are less robust but they show better performance for tracking (spatial resolutions $<100 \mu \mathrm{m}$ ) and for energy measurements (FWHM $\sim 25 \%$ ). New prototypes with customized resistivity of DLC foils are under construction, using an improved building technique that ensures the full coverage of current evacuation vias with pillar.

\section{Acknowledgments}

We would like to thank the CERN RD51 Collaboration for support and the CERN GDD Lab for MPGD tests.

\section{References}

[1] Y. Giomataris et al., MICROMEGAS: a high-granularity position-sensitive gaseous detector for high particle-flux environments, NIM Research Sec. A Vol. 376, Issue 1, 21 June 1996, pp. 29-35.

[2] T. Alexopoulos et al., A spark-resistant bulk-micromegas chamber for high-rate applications, NIM Research Sec. A Vol. 640, Issue 1, 1 June 2011, pp. 110-118.

[3] M. Alviggi et al., Pixelated resistive bulk micromegas for tracking systems in high rate environment, JINST Vol. 15, June 2020

[4] M. Alviggi et al., Small-pad resistive Micromegas for high rate environment: Performance of different resistive protection concepts, NIM Research Sec. A Vol. 936, Aug. 2019, pp. 408-41. 